\title{
Rentenbemessung in der schweizerischen gesetzlichen Unfallversicherung
}

\author{
Prof. Dr. iur. Thomas Gächter*
}

\section{Einleitende Bemerkungen}

\section{Keine Einschränkung auf Renten im engeren Sinn}

Da der Beginn der Rentenberechtigung nach einem Unfall in unterschiedlichen Rechtssystemen verschieden beschrieben wird, verbietet sich eine Darstellung, die den Gegenstand auf die Bemessung der eigentlichen Renten nach der Terminologie der schweizerischen Unfallversicherung einengt. Da bei fortdauernder Heilbehandlung oder Eingliederung über verhältnismässig lange Zeit hinweg Taggelder ausgerichtet werden können, ${ }^{1}$ sind auch die Grundzüge der Bemessung der Taggelder einzubeziehen, zumal sich diese hinsichtlich der Bemessung und Finanzierung nicht unerheblich von den Renten unterscheiden.

\section{Einschränkung auf Geldleistungen an die verunfallte Person}

Die Darstellung wird einzig die Geldleistungen behandeln, die der verunfallten Person selbst zukommen, d.h. Unfalltaggelder und Invalidenrenten der Unfallversicherung. Nicht behandelt werden die Geldleistungen an die Hinterlassenen einer tödlich verunfallten Person. Dabei ist jedoch anzumerken, dass sich diese hinsichtlich der Bemessung über weite Strecken an die Invalidenrente anlehnen. ${ }^{2}$

\section{Hauptpunkte der Darstellung}

Die folgende Darstellung wird einige Hauptpunkte in den Vordergrund rücken, die für die rechtsvergleichende Beurteilung des schweizerischen Systems der Unfallrentenbemessung von Interesse sein können:

\footnotetext{
* Frau lic. iur. Maya Geckeler Hunziker danke ich für die Sammlung von Material und die Ausarbeitung eines Teilentwurfs dieses Textes und Frau lic. iur. Eva Siki für die Durchsicht des Manuskripts.

1 Art. 16 Abs. 2 des Bundesgesetzes vom 20. März 1981 über die Unfallversicherung (UVG).

2 Art. 31 UVG.
} 
- Zunächst wird in einem kurzen Überblick über das historisch gewachsene Sozialversicherungssystem die Stellung und die Bedeutung der Unfallversicherung zu erläutern sein. Ohne diesen Hintergrund würden einige Besonderheiten in der Bemessung, insbesondere das Komplementärrentensystem, kaum verständlich.

- Von Interesse sind auch die Anspruchsvoraussetzungen für Geldleistungen, vor allem aber für Invalidenrenten der Unfallversicherung.

- Im Zentrum steht sodann die Frage nach den Bemessungsfaktoren der Taggelder und Renten.

- Insbesondere das schweizerische Komplementärrentensystem der Unfallversicherung kann einige Fragen aufwerfen.

- Einige Sonderprobleme ergeben sich systembedingt bei der Invalidität Teilzeiterwerbender. Da in der Schweiz Teilzeitarbeit ausserordentlich stark verbreitet ist, stehen diese Probleme in der Praxis häufig im Zentrum des Interesses.

\section{Stellung und Bedeutung der Unfallversicherung im schweizerischen So- zialversicherungssystem}

Die gesetzliche Unfallversicherung gehört historisch zu den ältesten Sozialversicherungszweigen der Schweiz. Sie wurde 1911 geschaffen, trat 1914 in Kraft und war zunächst nur für Arbeitnehmende in besonders gefahrgeneigten Berufen (Industrie und Transportwesen) obligatorisch (d.h. verpflichtend). Durchgeführt wurde die obligatorische Unfallversicherung von der Schweizerischen Unfallversicherungsanstalt (SUVA). Erst im Jahr 1984 wurde der obligatorische Versicherungsschutz auf sämtliche Arbeitnehmende ${ }^{3}$ in der Schweiz ausgedehnt. ${ }^{4}$ Seither sind alle Arbeitnehmenden für Arbeitsunfälle obligatorisch unfallversichert. Selbständigen steht der freiwillige Beitritt zur gesetzlichen Unfallversicherung offen. ${ }^{5}$

Mit der Schaffung des neuen Unfallversicherungsgesetzes wurde auch das bisherige Monopol der SUVA relativiert: Zwar verfügt diese für die ihr zugeordneten Betriebe nach wie vor über ein Monopol, ${ }^{6}$ doch wird die obligatorische gesetzliche Unfallversi-

3 Als Arbeitnehmerinnen und Arbeitnehmer gelten Personen, die in unselbständiger Stellung Arbeit leisten und dafür massgebenden Lohn nach dem jeweiligen Einzelgesetz beziehen (Art. 10 des Bundesgesetzes vom 6. Oktober 2000 über den Allgemeinen Teil des Sozialversicherungsrechts [ATSG]). Grundsätzlich gilt damit im schweizerischen System ein einheitlicher Arbeitnehmerbegriff. Die Qualifikation richtet sich dabei in der Regel nach der Statusfestlegung im Recht der Alters- und Hinterlassenenversicherung (siehe Art. 1 der Verordnung vom 20. Dezember 1982 über die Unfallversicherung [UVV]).

4 Art. 1a UVG.

5 Art. 4 UVG.

6 Art. 66 UVG i.V.m. Art. 73 ff. UVV. 
cherung für die anderen Betriebe durch andere Versicherer angeboten, wobei diese meist privatrechtlich organisiert sind. ${ }^{7}$

Im Gegensatz zur Alters-, Hinterlassenen- und Invalidenversicherung sowie zur gesetzlichen Krankenpflegeversicherung handelt es sich bei der gesetzlichen Unfallversicherung nicht um eine Volksversicherung für die gesamte Wohnbevölkerung, sondern um eine Klassenversicherung für Arbeitnehmende. Dies zeigt sich auch und insbesondere in der Art und im Umfang der Leistungen: Einerseits sind die von der Unfallversicherung erbrachten Sachleistungen vorteilhafter als diejenigen der sozialen Krankenpflegeversicherung (insbesondere unterliegen sie keinem Selbstbehalt), anderseits ist das Leistungsniveau der Geldleistungen vergleichsweise hoch, liegt doch der Entschädigungssatz für Taggelder und Invalidenrenten bei 80 Prozent des versicherten Verdienstes, ${ }^{8}$ wobei - zusammen mit anderen Rentenleistungen - sogar ein Entschädigungssatz von 90 Prozent erreicht werden kann, ${ }^{9}$ was im Wesentlichen einer vollen NettoEntschädigung entspricht.

\section{Besonderheiten der schweizerischen Unfallversicherung}

\section{Einschluss von Berufskrankheiten}

Wie andere Unfallversicherungssysteme schliesst auch die schweizerische Unfallversicherung die Versicherung gegen die Folgen von Berufskrankheiten mit ein, obwohl diese als Krankheiten im Sinn von Art. 3 ATSG gelten und damit grundsätzlich in den Bereich der Krankenpflegeversicherung fallen würden. ${ }^{10}$ Die Krankenversicherung wiederum ist erst seit 1996 für die gesamte Bevölkerung obligatorisch. Vorher war sie in der Regel als freiwillige Versicherung ausgestaltet, auf deren Abschluss der Unfallversicherungsgesetzgeber nicht abstellen konnte. ${ }^{11}$

7 Vgl. Art. 68 UVG.

8 Art. 17 und Art. 20 Abs. 1 UVG.

9 Art. 20 Abs. 2 UVG.

10 Art. 9 UVG.

11 Für die nicht der Unfallversicherung unterstehenden Personen erbringt die für die ganze Bevölkerung obligatorische Krankenversicherung die Sachleistungen im Zusammenhang mit der Krankenpflege. Geldleistungen wie Taggelder kennt die obligatorische Krankenversicherung indes nicht; die Krankentaggeldversicherung gilt zwar ebenfalls als Sozialversicherung (vgl. Art. 1a des Bundesgesetzes vom 18. März 1994 über die Krankenversicherung [KVG]), sie ist jedoch freiwillig. 


\section{Einschluss von Nichtberufsunfällen}

Als markante schweizerische Besonderheit kann gelten, dass die obligatorische Unfallversicherung von Anfang an auch die Nichtberufsunfälle der Versicherten abdeckte. Diese erstaunliche Ausweitung des Versicherungsschutzes einer Arbeitnehmerversicherung auf den Nichtberufsbereich rührt historisch vom Scheitern der so genannten „Lex Forrer" her. Diese umfassende Gesetzesvorlage sah eine obligatorische Unfall- und Krankenversicherung sowie die Militärversicherung vor, scheiterte im Jahr 1900 aber in der Volksabstimmung am Widerstand der bereits bestehenden Krankenversicherungen. In der Folge wurde eine separate Militärversicherung sowie, einige Jahre später, ein Kranken- und Unfallversicherungsgesetz geschaffen, das die Unfallversicherung als obligatorische Arbeitnehmerversicherung in gefahrgeneigten Betrieben strukturierte, die Krankenversicherung dagegen als freiwillige Versicherung, deren Leistungen aus öffentlichen Geldern teilweise subventioniert wurden. ${ }^{12} \mathrm{Um}$ mindestens die bereits obligatorisch Unfallversicherten zusätzlich abzusichern und damit einen Teilgehalt der „Lex Forrer" zu retten, wurde die obligatorische Nichtberufsunfallversicherung für die obligatorisch Unfallversicherten geschaffen. Wenn eine Arbeitnehmerin oder ein Arbeitnehmer der obligatorischen Versicherung unterstellt ist, spielt es mit anderen Worten für die Leistungen keine Rolle, ob sich ein Unfall während der Arbeitszeit oder ausserhalb der Arbeitszeit ereignet hat. Während jedoch jede unselbständige Erwerbstätigkeit der obligatorischen Berufsunfallversicherung untersteht, ist die Nichtberufsunfallversicherung nur obligatorisch, wenn die Tätigkeit einer Arbeitnehmerin oder eines Arbeitnehmers pro Woche acht Stunden überschreitet. ${ }^{13}$ Nur für Arbeitnehmerinnen und Arbeitnehmer, die unterhalb dieser zeitlichen Grenze beschäftigt sind, stellt sich somit etwa das Problem der Wegunfälle. Kraft ausdrücklicher Regelung gelten für diese Teilzeitbeschäftigten Wegunfälle als Unfälle im Sinn des Unfallversicherungsgesetzes. ${ }^{14}$

Die Prämien für die Berufsunfallversicherung werden vollständig vom Arbeitgeber getragen, jene für die Nichtberufsunfallversicherung gehen zu Lasten der Arbeitnehmenden. 15

Insbesondere bei der Anwendung des koordinierenden europäischen Sozialrechts, das auf der Grundlage des Personenfreizügigkeitsabkommens zwischen der Schweiz und der EU und ihren Mitgliedstaaten auch im Verhältnis zur Schweiz zur Anwendung gelangt, wird der atypische Charakter dieser Regelung spürbar: Während sich im vierten Kapitel der Verordnung 1408/71 einheitliche Regelungen für Arbeitsunfälle und Berufskrankheiten finden, müssen die Vorschriften zur Nichtberufsunfallsicherung - je

12 Siehe Hans Peter Tschudi, Entstehung und Entwicklung der schweizerischen Sozialversicherungen, Basel/Frankfurt a.M. 1989, S. 68 ff.

13 Art. 13 Abs. 1 UVV.

14 Art. 13 Abs. 2 UVV.

15 Art. 91 UVG. 
nach der jeweiligen Konstellation - in den Kapiteln über Krankheit oder Invalidität bzw. Alter- und Tod zusammengesucht werden.

\section{Unterschiedliche Finanzierungsverfahren von Taggeldern und Renten}

Die schweizerische Unfallversicherung erhält keine staatlichen Zuschüsse oder Subventionen. Sie finanziert sich ausschliesslich über Prämieneinnahmen, Regresseinnahmen und Zinserträge. ${ }^{16}$ Die Versicherer haben gesonderte Rechnungen für die obligatorische Versicherung der Berufsunfälle und Berufskrankheiten, für die obligatorische Versicherung der Nichtberufsunfälle und für die freiwillige Unfallversicherung zu führen, wobei die Finanzierung jedes Zweigs selbsttragend sein muss; ${ }^{17}$ d.h. die Prämien für alle drei Zweige müssen so bemessen sein, dass die Versicherungsleistungen selbsttragend finanziert werden können. ${ }^{18}$

Die Versicherer wenden kraft gesetzlicher Anordnung zur Finanzierung der Taggelder, der Kosten für die Heilbehandlung und der übrigen kurzfristigen Versicherungsleistungen das Ausgabenumlageverfahren an, d.h. die zu erbringenden Leistungen müssen durch Einnahmen des gleichen Jahres gedeckt sein. Zur Deckung aller Ausgaben aus bereits eingetretenen Unfällen sind angemessene Rückstellungen vorzunehmen. ${ }^{19}$

Zur Finanzierung der Invaliden- und Hinterlassenenrenten wenden sie dagegen das Rentenwertumlageverfahren an. ${ }^{20}$ Das durch dieses Verfahren geöffnete Deckungskapital muss für die Deckung aller Rentenansprüche aus bereits eingetretenen Unfällen ausreichen. Da das Verfahren eine Vorausfinanzierung künftiger Leistungen vorschreibt, handelt es sich begrifflich nicht um ein Umlageverfahren, sondern um eine Variante des Kapitaldeckungsverfahrens. ${ }^{21}$ Zusätzlich sind zum Ausgleich von Schwankungen der Betriebsergebnisse Reserven zu bestellen. ${ }^{22}$

16 Siehe Jean-Maurice Frésard/Margit Moser-Szeless, L'assurance-accidents obligatoire, in: Ulrich Meyer (Hrsg.), Soziale Sicherheit, Basel/Genf/München 2007, S. 825 ff., N. 593.

17 Art. 89 Abs. 2 und 3 UVG.

18 Mit anderen Worten sind Querfinanzierungen zwischen den einzelnen Zweigen, die vom selben Versicherer betrieben werden, nicht zulässig.

19 Art. 90 Abs. 1 UVG.

20 Art. 90 Abs. 2 UVG.

21 Alfred Maurer, Schweizerisches Unfallversicherungsrecht, 2. Aufl., Bern 1989, S. 571.

22 Art. 90 Abs. 4 UVG i.V.m. Art. 111 UVV. 


\section{Voraussetzung, Dauer und Bemessung von Taggeldern}

\section{Voraussetzungen, Beginn und Dauer}

Ist eine unfallversicherte Person aufgrund eines durch die Unfallversicherung abgedeckten Ereignisses voll oder teilweise arbeitsunfähig, so hat sie Anspruch auf ein Taggeld. ${ }^{23}$ Arbeitsunfähigkeit wird dabei definiert als durch eine Beeinträchtigung der körperlichen, geistigen oder psychischen Gesundheit bedingte, volle oder teilweise Unfähigkeit, im bisherigen Beruf oder Aufgabenbereich zumutbare Arbeit zu leisten, wobei bei langer Dauer auch die zumutbare Tätigkeit in einem anderen Beruf oder Aufgabenbereich zu berücksichtigen ist. ${ }^{24}$

Der Anspruch auf Taggeld entsteht am dritten Tag nach dem Unfalltag, d.h. es besteht von Gesetzes wegen eine Wartefrist von zwei Arbeitstagen. Er erlischt mit der Wiedererlangung der vollen Arbeitsfähigkeit, mit dem Beginn einer Rente oder mit dem Tod des Versicherten. ${ }^{25}$ Taggelder werden für alle Tage, einschliesslich der Sonn- und Feiertage, ausgerichtet. ${ }^{26}$ Eine maximale Taggeldzahl ist nicht vorgesehen, d.h. eine versicherte Person kann auch über Jahre hinweg Taggelder beziehen, sofern die Arbeitsunfähigkeit andauert und von der Fortsetzung der ärztlichen Behandlung nach wie vor eine namhafte Besserung des Gesundheitszustands erwartet werden kann. ${ }^{27}$

\section{Versicherter Verdienst, Entschädigungssatz}

Taggelder und Renten werden nach dem versicherten Verdienst bemessen, wobei die Berechnungsbasis des versicherten Verdienstes bei Taggeldern eine andere ist als bei Renten: Als versicherter Verdienst gilt für die Bemessung der Taggelder der letzte vor dem Unfall bezogene Lohn, einschliesslich der noch nicht ausbezahlten Lohnbestandteile, auf die ein Rechtsanspruch besteht. Bei Renten dagegen ist grundsätzlich der innerhalb eines Jahres vor dem Unfall bezogene Lohn massgeblich. ${ }^{28}$

Der versicherte Verdienst aus unselbständiger Erwerbstätigkeit wird jedoch, ähnlich wie in den meisten Unfallversicherungssystemen, auf einen Höchstbetrag begrenzt. Er ist nach der Vorgabe des Gesetzes so anzusetzen, dass mindestens 92 Prozent, aber nicht mehr als 96 Prozent der versicherten Arbeitnehmer zum vollen Verdienst versi-

23 Art. 16 Abs. 1 UVG.

24 Art. 6 ATSG.

25 Art. 16 Abs. 2 UVG.

26 Art. 25 Abs. 1 UVV.

27 Art. 19 Abs. 1 UVG e contrario.

28 Art. 15 Abs. 2 UVG. 
chert sind. ${ }^{29}$ Gegenwärtig beläuft sich der Höchstbetrag auf 126'000 Franken im Jahr oder 346 Franken pro Tag, d.h. auf rund 79'202 Euro jährlich oder 217 Euro täglich. 30

In einigen Sonderfällen wird der massgebende Lohn für die Berechnung der Taggelder in spezieller Weise bestimmt, so etwa bei Versicherten, die wegen Militär-, Ziviloder Zivilschutzdienst, Unfall, Krankheit, Mutterschaft oder Kurzarbeit keinen oder einen verminderten Lohn bezogen haben. Bei diesen Personengruppen wird der Verdienst berücksichtigt, den sie ohne den Eintritt der genannten Umstände erzielt hätten. ${ }^{31}$ Zudem wird bei unregelmässig Beschäftigten oder bei Beschäftigten mit stark schwankendem Lohn auf einen durchschnittlichen Tagesverdienst abgestellt. ${ }^{32}$

Das Taggeld beträgt bei voller Arbeitsunfähigkeit 80 Prozent des versicherten Verdienstes und wird bei teilweiser Arbeitsunfähigkeit entsprechend gekürzt. ${ }^{33}$

\section{Taggeldberechnung}

Die Berechnungsformel für die Taggelder ergibt sich aus einem speziellen Anhang zur Unfallversicherungsverordnung. Nach der in diesem Anhang enthaltenen Formel berechnet sich das Taggeld wie folgt: Versicherter Jahresverdienst geteilt durch 365, multipliziert mit 80 Prozent. Der Jahresverdienst berechnet sich dabei durch die Multiplikation des letzten vor dem Unfall bezogenen Monatslohns mit zwölf, inkl. der speziell bezeichneten Lohnbestandteile und der noch nicht ausbezahlten Lohnansprüche. Maximal beträgt das Taggeld damit - ausgehend vom Höchstbetrag des versicherten Verdienstes - 277 Franken, d.h. rund 174 Euro. Bei teilweiser Arbeitsunfähigkeit wird das Taggeld, wie gesagt, entsprechend anteilsmässig gekürzt.

Kurz zusammengefasst sind die massgeblichen Faktoren für die Berechnung des Taggelds damit der Grad der Arbeitsunfähigkeit, der auf der Grundlage des letzten Monatslohns berechnete versicherte Verdienst bis zur Höchstgrenze und der Entschädigungssatz von 80 Prozent.

29 Art. 15 Abs. 3 UVG.

30 Art. 22 Abs. 1 UVV. Bis Ende Dezember 2007 belief sich dieser Betrag während acht Jahren auf 106'800 Franken.

31 Art. 23 Abs. 1 UVV.

32 Art. 23 Abs. 3 UVV. Für weitere Personengruppen wie Praktikanten und Volontäre sowie bei der unfallbedingten Verlängerung von Ausbildungen kennt das Verordnungsrecht weitere Spezialregelungen (siehe Art. 23 Abs. 4-9 UVV).

33 Art. 17 Abs. 1 UVG. 


\section{Zusammentreffen mit Taggeldern anderer Sozialversicherungen}

Das Taggeld der Unfallversicherung wird nicht gewährt, wenn ein Anspruch auf ein Taggeld der Invalidenversicherung oder eine Mutterschaftsentschädigung nach dem Erwerbsersatzgesetz besteht. ${ }^{34}$

\section{Invalidenrenten der Unfallversicherung}

\section{Voraussetzungen und Dauer des Rentenanspruchs}

\section{a) Grundlagen}

Ist die versicherte Person infolge des Unfalls zu mindestens 10 Prozent invalid i.S. von Art. 8 ATSG, so hat sie Anspruch auf eine Invalidenrente. ${ }^{35}$ Massgeblich ist auch für die Unfallversicherung der allgemeine rentenrelevante Invaliditätsbegriff, wie er im Bundesgesetz über den Allgemeinen Teil des Sozialversicherungsrechts (ATSG) definiert wird. Laut Art. 8 Abs. 1 ATSG besteht Invalidität in der voraussichtlich bleibenden oder längere Zeit dauernden ganzen oder teilweisen Erwerbsunfähigkeit. Als Erwerbsunfähigkeit wiederum wird der durch Beeinträchtigung der körperlichen, geistigen oder psychischen Gesundheit verursachte und nach zumutbarer Behandlung und Eingliederung verbleibende ganze oder teilweise Verlust der Erwerbsmöglichkeiten auf dem in Betracht kommenden ausgeglichenen Arbeitsmarkt definiert. ${ }^{36}$

Es gehört damit zu den Grundtheoremen des schweizerischen Invalidenleistungssystems, dem auch die Invalidenrenten der Unfallversicherung zuzurechnen sind, dass die Rente nicht dem Ersatz der körperlichen Beeinträchtigung oder der Abgeltung seelischer Unbill der verunfallten Person dient, sondern einzig dem Ersatz des unfallbedingten Erwerbsausfalls. Trotz erheblicher Beeinträchtigungen auf dem übrigen Arbeitsmarkt muss also etwa ein Rechtsanwalt oder Buchhalter, der durch einen Unfall zum Paraplegiker wird, nicht unbedingt auch als (in rentenrelevanter Weise) invalid im Sinn der Invaliden- oder Unfallversicherung gelten: So lange er nach erfolgter (Wieder-) Eingliederung nicht weniger Einkommen erzielt, als er ohne den entsprechenden Gesundheitsschaden erzielen würde, besteht keine Beeinträchtigung der Erwerbsfähigkeit und damit auch keine Invalidität.

Die Genugtuungs- oder Schmerzensgeldfunktion übernimmt im schweizerischen Unfallversicherungssystem die Integritätsentschädigung, die einer verunfallten versicherten Person zusteht, wenn sie eine dauernde erhebliche Schädigung der körperlichen, geisti-

\footnotetext{
34 Art. 16 Abs. 3 UVG.

35 Art. 18 Abs. 1 UVG.

36 Art. 7 ATSG.
} 
gen oder psychischen Integrität erlitten hat. ${ }^{37}$ Die Integritätsentschädigung wird in Form eine Kapitalleistung gewährt, darf den am Unfalltag geltenden Höchstbetrag des versicherten Jahresverdienstes nicht übersteigen und wird entsprechend der Schwere des Integritätsschadens abgestuft. 38 Maximal kann damit gegenwärtig eine (einmalige) Integritätsentschädigung von 126'000 Franken erbracht werden; dies aber nur in ganz gravierenden Fällen, so etwa bei unfallbedingter Tetraplegie oder unfallbedingter vollständiger Blindheit. Für geringfügigere Beeinträchtigungen wie beispielsweise den Verlust einer Hand oder den Verlust einer Niere werden Entschädigungen in der Höhe von 40 bzw. 20 Prozent dieses Maximalbetrags erbracht. ${ }^{39}$ Es ist also denkbar, dass jemand, der unfallbedingt eine Hand verliert, Anspruch auf eine einmalige Integrationsentschädigung von rund 50'400 Franken hat, aufgrund vollständiger weiterer Erwerbsfähigkeit aber nicht als invalid gilt und damit keine Rentenleistungen der Unfallversicherung erhält.

Anders als bei der Arbeitsunfähigkeit, welche die Voraussetzung für die Ausrichtung von Taggeldern bildet, beurteilt sich die Erwerbsunfähigkeit nach der Vornahme der zumutbaren Behandlung oder Eingliederung. Bei der Erwerbsunfähigkeit, und damit auch beim Invaliditätsbegriff der Unfallversicherung, handelt es sich um einen wirtschaftlichen Begriff, da sie wirtschaftliche Möglichkeiten und nicht gesundheitliche Beeinträchtigungen beurteilt. ${ }^{40}$ Mit dem Kriterium des ,ausgeglichenen Arbeitsmarkts“ soll die Abgrenzung zu allenfalls konjunkturell bedingter Arbeitslosigkeit ermöglicht werden. ${ }^{41}$

\section{b) Voraussetzungen des Anspruchs}

Der Rentenanspruch entsteht, wenn von der Fortsetzung der ärztlichen Behandlung keine namhafte Besserung des Gesundheitszustandes des Versicherten mehr erwartet werden kann und allfällige Eingliederungsmassnahmen der Invalidenversicherung abgeschlossen sind. Mit dem Rentenbeginn fallen die Heilbehandlung und die Taggeldleistungen dahin. 42

Ist von der Fortsetzung der ärztlichen Behandlung keine namhafte Besserung des Gesundheitszustandes der versicherten Person mehr zu erwarten, wird jedoch der Entscheid der Invalidenversicherung über die berufliche Eingliederung erst später gefällt, so wird vom Abschluss der ärztlichen Behandlung an vorübergehend eine Rente ausge-

37 Art. 24 Abs. 1 UVG.

38 Art. 25 Abs. 1 UVG.

39 Siehe den Anhang 3 zur UVV.

40 Z.B. Thomas Locher, Grundriss des Sozialversicherungsrechts, 3. Aufl., Bern 2003, S. 121. Im Gegensatz zur Arbeitsunfähigkeit, die sich auch auf den bisherigen „Aufgabenbereich“ bezieht (also auch auf Nichterwerbstätige), bezieht sich die Erwerbsunfähigkeit nur auf erwerbliche Tätigkeiten.

41 Siehe Locher (Fn. 40), S. 124.

42 Art. 19 Abs. 1 UVG. 
richtet ${ }^{43}$; diese wird aufgrund der in diesem Zeitpunkt bestehenden Arbeitsunfähigkeit festgesetzt. Der Anspruch auf diese Übergangsrente erlischt beim Beginn des Anspruchs auf ein Taggeld der Invalidenversicherung, mit dem negativen Entscheid der Invalidenversicherung über die berufliche Eingliederung oder mit der Festsetzung der definitiven Rente. ${ }^{44}$

\section{c) Dauer der Rentenleistung 45}

Der Versicherer muss bei der Festsetzung der Invalidenrente eine Prognose über den künftigen Verlauf der Erwerbsunfähigkeit stellen. Wenn er von vornherein mit einer bleibenden Erwerbsunfähigkeit rechnet, setzt er eine Dauerrente fest. Die UnfallInvalidenrente wird grundsätzlich bis ans Lebensende gewährt. Sie wird nicht durch allenfalls tiefere Altersleistungen abgelöst. Damit erfüllt die Unfallversicherung auch die Funktion einer Alterssicherung und gehört für die Leistungen ab dem ordentlichen Pensionierungsalter materiell zur Alters- und Hinterlassenenvorsorge. Sie gleicht damit den Umstand aus, dass eine invalide Person den entsprechenden Vorsorgeschutz nicht angemessen ausbauen konnte.

Freilich kann auch eine Dauerrente geändert oder sogar aufgehoben werden, wenn später ein Revisionsgrund gemäss Art. 17 ATSG festgestellt wird. Eine Rentenrevision ist erforderlich, wenn sich der Invaliditätsgrad erheblich verändert. In der Invalidenversicherung und in der beruflichen Vorsorge ist die Rentenabstufung verhältnismässig grob in Viertelsrenten, halbe Renten und ganze Renten gegliedert. Erheblich ist in diesen Systemen erst eine Veränderung, die sich auf die Rentenhöhe auswirken würde. Die Unfallrenten werden dagegen prozentgenau nach Massgabe des Invaliditätsgrads ausgerichtet. Praxisgemäss gelten dabei Änderungen von 5 Prozent unterhalb eines Invaliditätsgrads von 50 Prozent als erheblich, bei höheren Invaliditätsgraden werden für die Bejahung der Erheblichkeit Änderungen von 10 Prozent verlangt. ${ }^{46}$ Die Revision kann grundsätzlich jederzeit von Amts wegen oder auf Gesuch hin vorgenommen werden. Aufgrund ihrer soeben beschriebenen Zusatzfunktion in der Alterssicherung ist die Möglichkeit der Rentenrevision indes - in Abweichung von Art. 17 ATSG - eingeschränkt: Ab dem 65. Altersjahr kann bei Männern und ab dem 62. Altersjahr bei Frauen die Unfallrente nicht mehr revidiert werden (Art. 22 UVG).

Wenn der Unfallversicherer bei der Rentenfestsetzung die Prognose stellt, dass sich der Versicherte in absehbarer Zeit mit Wahrscheinlichkeit an die Unfallfolgen gewöhnen und anpassen wird, gewährt er ihm die Invalidenrente nur für eine bestimmte Zeit,

43 Siehe auch Art. 19 Abs. 3 UVG i.V.m. Art. 30 Abs. 1 UVV.

44 Art. 30 Abs. 1 UVV.

45 Zum Ganzen Abschnitt Alfred Maurer, Bundessozialversicherungsrecht, 2. Aufl., Basel/Frankfurt a.M. 1994, S. 371 ff., z.T. wörtlich.

46 Siehe Ueli Kieser, Bundesgesetz über den Allgemeinen Teil des Sozialversicherungsrechts (ATSG), in: Ulrich Meyer (Hrsg.), Soziale Sicherheit, Basel/Genf/München 2007, S. 217 ff., N. 43. 
z.B. für drei Jahre. Die Möglichkeit der befristeten Rente ist im Gesetz zwar nicht ausdrücklich vorgesehen, von der Praxis aber anerkannt. Der Versicherte kann sich darauf einstellen, dass die Rente nach Ablauf dieser Zeit automatisch dahinfällt. Der Richter darf nur prüfen, ob die für die Befristung erforderliche Prognose sachgerecht, z.B. gestützt auf überzeugende medizinische Gutachten, gestellt worden ist.

Bei einfachen typischen Verletzungen lässt sich voraussehen, dass die Erwerbsunfähigkeit des Versicherten zufolge Angewöhnung und Anpassung allmählich abnimmt. Diesem Umstand trägt der Versicherer gewöhnlich Rechnung, indem er die Rente von vornherein abstuft und sie z.B. für je ein Jahr entsprechend einer Erwerbsunfähigkeit von 40, 30 und 20 Prozent festsetzt. Die Abstufung ist bei Zeit- und bei Dauerrenten zulässig. Mit ihr soll der Wille des Versicherten, sich an die Unfallfolgen anzupassen und zu gewöhnen, gestärkt werden. Wenn sich die Prognose im Nachhinein als unrichtig erweist, wird in einer Revisionsverfügung von der Abstufung abgesehen oder wird die Rente angepasst.

Bei verhältnismässig geringen Invalidenrenten, d.h. bei solchen, die den Betrag von gegenwärtig rund 173 Franken pro Monat nicht übersteigen, kann der Versicherer die Rente jederzeit nach ihrem Barwert auskaufen. In den übrigen Fällen ist der Auskauf nur mit dem Einverständnis und im offenkundigen langfristigen Interesse des Rentenberechtigten zulässig. 47

\section{Normal- und Komplementärrente}

\section{a) Normalrente}

Die Höhe einer normalen Invalidenrente der Unfallversicherung bestimmt sich denkbar einfach: Sie beträgt bei Vollinvalidität 80 Prozent des versicherten Verdienstes, bei Teilinvalidität wird sie entsprechend gekürzt. ${ }^{48}$ Der Fall einer eigentlichen Normalrente tritt aber eher selten ein: Nur bei Invaliditätsgraden zwischen 10 Prozent, durch welche in der Unfallversicherung bereits Rentenansprüche begründet werden, und 40 Prozent, durch die Rentenansprüche der Invalidenversicherung ausgelöst werden. ${ }^{49}$ Das heisst, dass in der Regel nur bei Teilrenten im unteren Bereich „Normalrenten“ ausbezahlt werden.

47 Art. 35 Abs. 1 UVG.

48 Art. 20 Abs. 1 UVG.

49 Siehe Art. 28 Abs. 1 des Bundesgesetzes vom 19. Juni 1959 über die Invalidenversicherung (IVG). 


\section{b) Komplementärrente}

Da der Invaliditätsbegriff im Sinn von Art. 8 ATSG - nicht aber unbedingt auch die Bemessung des Invaliditätsgrads 50 - in der Unfallversicherung und in der Invalidenversicherung übereinstimmen, treffen in der überwiegenden Zahl der Fälle Renten der Unfallversicherung mit solchen der Invaliden- oder, bei älteren Erwerbstätigen, der Altersund Hinterlassenenversicherung zusammen, allenfalls auch mit solchen der beruflichen Vorsorge.

Wird eine Person aus gesundheitlichen Gründen erwerbsunfähig, hat sie zunächst Anspruch auf eine Rente der Invalidenversicherung, sofern der Invaliditätsgrad mindestens 40 Prozent beträgt. Unter den gleichen Voraussetzungen kann sie eine Invalidenrente der beruflichen Vorsorge beanspruchen, sofern sie den BVG-Mindestlohn erreicht. 51

Art. 66 Abs. 1 und 2 ATSG legen fest, dass die Renten unter Vorbehalt einer Überentschädigung kumulativ und in folgender Reihenfolge gewährt werden: Invalidenversicherung, Unfallversicherung und Militärversicherung, berufliche Vorsorge.

In diesen Fällen wird der versicherten Person seitens der Unfallversicherung eine so genannte Komplementärrente gewährt. Diese entspricht - in Abweichung der allgemeinen Überentschädigungsgrenze von Art. 69 ATSG - der Differenz zwischen 90 Prozent des versicherten Verdienstes und der Rente der IV oder der AHV, höchstens aber dem für Voll- oder Teilinvalidität vorgesehenen Betrag. 52

Mit anderen Worten: Die Normalrente mit einem Entschädigungssatz von 80 Prozent des versicherten Verdienstes ist der atypische Fall bei geringerer unfallbedingter Invalidität, die Komplementärrente mit einem Entschädigungssatz von (zusammen mit der Rente der Alters-, Hinterlassenen und Invalidenversicherung) insgesamt 90 Prozent des versicherten Verdienstes bildet den Hauptfall der Unfallrente.

50 Dies ergibt sich daraus, dass die Invalidenversicherung auch die Einschränkung im nichterwerblichen Bereich absichert und bei Teilerwerbstätigen damit die so genannte gemischte Methode der Invaliditätsgradbemessung zur Anwendung kommt (siehe Art. 28 Abs. 2ter IVG, ab 1. Januar 2008 Art. 28a Abs. 3 IVG).

51 Art. 7 des Bundesgesetzes vom 25. Juni 1982 über die berufliche Alters-, Hinterlassenen- und Invalidenvorsorge (BVG) und Art. 5 der Verordnung vom 18. April 1984 über die berufliche Alters-, Hinterlassenen- und Invalidenvorsorge (BVV 2).

52 Art. 20 Abs. 2 UVG. 


\section{Bemessungsgrundlagen}

a) Unterschiedliche Bemessungsgrundlagen für Renten der Unfallversicherung, der Invalidenversicherung und der beruflichen Vorsorge

Für die Höhe der Unfallversicherungsrente ist neben dem Invaliditätsgrad der unmittelbar vor dem Unfall erzielte Lohn von entscheidender Bedeutung. In der Unfallversicherung beträgt die Unfallrente bei Vollinvalidität 80 Prozent des innerhalb eines Jahres vor dem Unfall bezogenen Lohns. ${ }^{53}$ Die Unfallversicherungsrente ist damit eine auf eine einjährige Referenzperiode bezogene Risikoleistung.

In der Invalidenversicherung und in der beruflichen Vorsorge kommt es dagegen auf den Lohn an, der während des gesamten bisherigen Erwerbslebens erzielt worden ist. In der Invalidenversicherung richtet sich die Höhe der Rente neben dem Invaliditätsgrad nach der Beitragsdauer und der Höhe der insgesamt einbezahlten Lohnbeiträge. Wer keine Beitragslücken aufweist, erhält bei einem Invaliditätsgrad von mindestens 70 Prozent eine monatliche Rente von zwischen 1'105 und 2'210 Franken, je nach Höhe der einbezahlten Beiträge. ${ }^{54}$

In der beruflichen Vorsorge hängt die Rentenhöhe neben dem Invaliditätsgrad vom Altersguthaben und dem Umwandlungssatz ab. Das Altersguthaben umfasst die verzinsten Altersgutschriften ${ }^{55}$, die eine versicherte Person bis zum Eintritt der Invalidität erworben hat, sowie die (fiktiven) Altersgutschriften für die bis zum Rentenalter fehlenden Jahre ohne Zinsen. ${ }^{56}$ Multipliziert man das so bestimmte Altersguthaben mit dem Umwandlungssatz von 6,8 Prozent, ergibt sich der jährliche Betrag einer vollen Invalidenrente. 57

\section{b) Invaliditätsgrad}

Der Invaliditätsgrad bestimmt sich, wie bereits beschrieben, nach der allgemeinen Methode des Einkommensvergleichs gemäss Art. 16 ATSG. Nach dieser Methode wird das Erwerbseinkommen, das die versicherte Person nach Eintritt der Invalidität und

53 Art. 20 Abs. 1 i.V.m. Art. 15 Abs. 2 UVG.

54 Art. 36 Abs. 2 und Art. 37 Abs. 1 IVG i.V.m. Art. 29 ff. des Bundesgesetzes vom 20. Dezember 1946 über die Alters- und Hinterlassenenversicherung (AHVG) und Art. 50 ff. der Verordnung vom 31. Oktober 1947 über die Alters- und Hinterlassenenversicherung (AHVV).

55 Die Altersgutschriften, welche die Grundlage für die Berechnung des Altersguthabens bilden, werden jährlich in Prozenten des koordinierten Lohns berechnet. Sie betragen gegenwärtig zwischen sieben Prozent für die Altersgruppe der zwischen 25- und 44-Jährigen und 18 Prozent für die Altersgruppe von 55 bis 65 Jahren (Art. 16 BVG). Diese Gutschriften sind jedoch nicht mit den effektiven Beitragssätzen zu verwechseln, die aufgrund reglementarischer Bestimmungen der Vorsorgeeinrichtungen festgelegt werden.

56 Art. 24 Abs. 2 und 3 BVG.

57 Art. 14 Abs. 2 i.V.m. Art. 24 Abs. 2 BVG. 
nach Durchführung der medizinischen Behandlung und allfälliger Eingliederungsmassnahmen durch eine ihr zumutbare Tätigkeit bei ausgeglichener Arbeitsmarktlage erzielen könnte (Invalideneinkommen), in Beziehung gesetzt zum Erwerbseinkommen, das sie erzielen könnte, wenn sie nicht invalid geworden wäre (Valideneinkommen). ${ }^{58}$ Ein rechnerisch exakt erzieltes Ergebnis wird sodann nach den Regeln der Mathematik auf die nächste ganze Prozentzahl auf- oder abgerundet. 59

Aus dem Wortlaut „erzielen könnte“ in Art. 16 ATSG ergibt sich, dass sowohl das Validen- als auch das Invalideneinkommen hypothetisch zu ermitteln ist. Für die Bestimmung des Valideneinkommens wird dabei jedoch durchwegs das vor dem Eintritt der zur Invalidität führenden Arbeitsunfähigkeit erzielte Einkommen gewählt, wobei dieses den bis zum Zeitpunkt der Invaliditätsbemessung eingetretenen Entwicklungen anzupassen ist. Damit wird dem Postulat genügt, nach welchem die Vergleichseinkommen so konkret wie möglich zu bestimmen sind. ${ }^{60}$ Zur Bestimmung des Invalideneinkommens greift die Praxis in aller Regel auf Tabellenwerte zurück. Besonderes Gewicht hat dabei die vom Bundesamt für Statistik erarbeitete Lohnstrukturerhebung. Die Werte werden allerdings in verschiedener Hinsicht korrigiert, weil die konkreten Besonderheiten wie die nur noch beschränkte Einsatzfähigkeit einer Person zu berücksichtigen sind. Praxisgemäss dürfen solche Abzüge zusammen aber nicht mehr als 25 Prozent des ermittelten Invalideneinkommens ausmachen. ${ }^{61}$ Die Rechtsprechung hat - unter restriktiven Voraussetzungen - auch zugelassen, dass Versicherer für die Bestimmung des Invalidenlohns auf (eigene) Datensammlungen abstellen können. ${ }^{62}$ Wenn diese qualitativ und quantitativ genügen, können sie der konkreten Ermittlung des Invalideneinkommens dienlich sein. So hat etwa die SUVA innert der letzten zwölf Jahre unter dem Titel DAP (Dokumentation von Arbeitsplätzen) eine spezielle Datenbank aufgebaut. Schweizweit werden durch speziell geschulte Aussendienstmitarbeiter konkrete Arbeitsplätze erfasst und dokumentiert. Die Arbeitsplatzbeschreibung erfasst die Region, die Ausbildungsanforderungen, die Arbeitszeit, die Eignung für Männer oder Frauen, andere äussere Einflüsse, den gesamten Arbeitsablauf und die Verdienstverhältnisse. ${ }^{63}$

58 Siehe z.B. Ueli Kieser, Kommentar ATSG, Zürich/Basel/Genf 2003, Art. 16 N. 6. Konkret errechnet sich der Invaliditätsgrad nach der Formel: Valideneinkommen abzüglich Invalideneinkommen multipliziert mit 100 und sodann geteilt durch das Valideneinkommen. Bei einem Valideneinkommen von 100'000 Franken und einen Invalideneinkommen von 25'000 Franken würde sich deshalb ein Invaliditätsgrad von 75 Prozent ergeben.

59 BGE $130 \mathrm{~V} 121$.

60 Kieser (Fn. 58), Art. 16 N. 11.

61 Kieser (Fn. 58), Art. 16 N. 13.

62 BGE $129 \mathrm{~V} 472 \mathrm{ff}$.

63 Zum Ganzen Stefan A. Dettwiler, Suva „DAP“t nicht im Dunkeln. Invalidenlohnbemessung anhand konkreter Arbeitsplätze (DAP), Schweizerische Zeitschrift für Sozialversicherung und berufliche Vorsorge (SZS) 2006, S. 6 ff. 


\section{c) Versicherter Verdienst im Jahr vor dem Unfall}

Der Rentenbemessung wird der innerhalb eines Jahres vor dem Unfall bezogene Lohn zu Grunde gelegt. ${ }^{64}$ Maßgeblich ist der in dieser Zeitspanne tatsächlich bezogene Lohn, nicht ein möglicherweise zukünftig erreichbarer Lohn. Der Versicherte kann sich also nicht auf einen hypothetischen Verdienst in seinem angestammten Beruf berufen, den er (beispielsweise wegen einer wirtschaftlichen Krisenlage) nicht ausgeübt hat, vielmehr ist der bei der tatsächlich ausgeübten Tätigkeit erzielte Verdienst maßgebend.

Zur Bemessung des versicherten Verdienstes wird grundsätzlich auf den in der AHV maßgebenden Lohn abgestellt. Für die in Art. 22 Abs. 2 UVV genannten Fälle wird jedoch vom maßgebenden Lohn abgewichen:

- So gelten Löhne, auf denen wegen des Alters des Versicherten keine Beiträge der AHV erhoben werden, als versicherter Verdienst (lit. a).

- Zum versicherten Verdienst zählen auch die Familienzulagen, welche als Kinder-, Ausbildungs- oder Haushaltszulagen im orts- oder branchenüblichen Rahmen gewährt werden (lit. b). Durch den Einbezug dieser Leistungen in die Berechnung des versicherten Verdienstes erklärt sich, weshalb Bezügerinnen und Bezüger einer UV-Rente keine zusätzlichen Leistungen für ihre Familienlasten erhalten. Durch den Einbezug dieser Leistungen in den versicherten Verdienst werden sie bereits hinreichend berücksichtigt.

- Lit. c enthält eine Sonderregelung für „mitarbeitende Familienglieder“. Danach bildet der berufs- und ortsübliche Lohn Grundlage der UV-Rente. Mit dieser Bestimmung soll eine Benachteiligung der Familienmitglieder mit nicht arbeitsmarktkonformen Löhnen verhindert werden, so dass sie nur dann zur Anwendung gelangt, wenn die ausgerichteten Löhne auf Grund der Familienzugehörigkeit tiefer ausfallen als berufs- und ortsüblich.

- Schließlich werden Entschädigungen bei Auflösung des Arbeitsverhältnisses, bei Betriebsschließungen, Betriebszusammenlegungen oder bei ähnlichen Gelegenheiten bei der Bestimmung des versicherten Verdiensten nicht mitberücksichtigt (lit. d).

Dauerte das Arbeitsverhältnis noch nicht das ganze Jahr, so werden die in dieser Zeit bezogenen Verdienste auf ein volles Jahr umgerechnet. ${ }^{65}$ Dabei wird bei unterjährigen Arbeitsverhältnissen vermutet, dass der Versicherte ganzjährig zu den gleichen Bedingungen gearbeitet hätte, weshalb die Umrechnung nach Art. 22 Abs. 4 Satz 2 UVV auf zwölf Monate erfolgt. Bei Versicherten, die nur einen zeitlich begrenzten Teil des Jahrs erwerbstätig sind, erfolgt keine Umrechnung, sondern es gilt der Verdienst während der vereinbarten Dauer, wie etwa bei Studierenden und Schülern, die nur während der Ferien arbeiten. Dies gilt auch für unregelmäßig Beschäftigte oder bei einer befristeten Beschäftigung. Im Gegensatz dazu wird bei einer unüblichen Reduktion der Arbeitszeit

64 Art. 15 Abs. 2 UVG.

65 Satz 2 von Art. 22 Abs. 4 UVV. 
mit Ausnahmecharakter wie beispielsweise einem einmaligen unbezahlten Urlaub, der versicherte Verdienst i.S. von Art. 22 Abs. 4 Satz 2 UVV ergänzt. Die Umrechnung des Lohns i.S. von Art. 22 Abs. 4 Satz 2 UVV ist mithin nicht auf Fälle beschränkt, in welchen das Arbeitsverhältnis bis zum Unfall noch kein ganzes Jahr gedauert hat.

\section{d) Versicherter Verdienst in Sonderfällen}

Hat der Versicherte im Jahr vor dem Unfall wegen Militärdienst, Zivildienst, Zivilschutzdienst, Unfall, Krankheit, Mutterschaft, Arbeitslosigkeit oder Kurzarbeit einen verminderten Lohn bezogen, so wird der versicherte Verdienst nach dem Lohn festgesetzt, den der Versicherte ohne diese Absenz erzielt hätte (Art. 24 Abs. 1 UVV).

Beginnt die Rente mehr als fünf Jahre nach dem Unfall, so ist der Lohn maßgebend, den der Versicherte ohne Unfall im Jahr vor Rentenbeginn bezogen hätte, sofern er höher ist als der letzte vor dem Unfall erzielte Lohn. Diese Bestimmung bezweckt die Anpassung der Rente an die normale Lohnentwicklung 66 im angestammten Tätigkeitsbereich. ${ }^{67}$ Andere den versicherten Lohn beeinflussende Änderungen in den erwerblichen Verhältnissen werden dabei aber nicht berücksichtigt. ${ }^{68}$ Bei einem Versicherten, der wie alle anderen Angestellten mit überwiegender Wahrscheinlichkeit ohne Unfall weiterhin Überstundenarbeit geleistet hätte und somit im Jahr vor dem Rentenbeginn ein höheres Einkommen erzielen würde als vor dem Unfall, ist der versicherte Verdienst i.S. von Art. 24 Abs. 2 UVV anzupassen. 69

Bezog der Versicherte wegen beruflicher Ausbildung am Tag des Unfalls nicht den Lohn eines Versicherten mit voller Leistungsfähigkeit derselben Berufsart, so wird der versicherte Verdienst von dem Zeitpunkt an, da er die Ausbildung abgeschlossen hätte, nach dem Lohn festgesetzt, den er im Jahr vor dem Unfall als voll Leistungsfähiger erzielt hätte. Nach dem klaren Wortlaut dieser Bestimmung muss die Entlöhnung der Tätigkeit wegen der Ausbildung niedriger sein als der Lohn des voll Leistungsfähigen derselben Berufsart. Die berufliche Ausbildung selbst muss also für den kleineren, berufsunüblichen Lohn kausal sein. Außerdem muss es sich bei den Erwerbszweigen des in Ausbildung Stehenden und des bereits Ausgebildeten um dieselbe Berufsart handeln. Somit ist Art. 24 Abs. 3 UVV auf einen Werkstudenten, der im Sommer im Baugewer-

66 Vgl. BGE 123 V 51 E. 3c.

67 RKUV 1993 Nr. U 161 S. 52 E. 3b; RKUV 1999 Nr. U 327 S. 111 f. E. 3c.

68 BGE 127 V 172 E. 3b. Die Bestimmung soll jedoch versicherte Personen nicht durch Berücksichtigung individueller Lohnentwicklungen (z.B. Hinzutreten von Kinderzulagen) gegenüber solchen besser stellen, deren Rente innert fünf Jahren nach dem Unfall festgesetzt wurde (BGE 127 V 173 E. 3b). Bei der Bestimmung des versicherten Jahresverdienstes ist deshalb auch bei einem Rentenbeginn mehr als fünf Jahre nach dem Unfall auf die im Unfallzeitpunkt ausgeübte Tätigkeit abzustellen (RKUV 1999 Nr. U 340 S. 405 E. 3c).

69 RKUV 2000 Nr. U 400 S. 381 ff. E. 2 b und c. 
be als Hilfsmonteur arbeitet, nicht anwendbar, weil die Tätigkeit des Bauarbeiters nicht in Verbindung mit dem angestrebten Berufsziel eines Biologielehrers steht. 70,71

Bei Bezug einer Invalidenrente bestimmt sich der versicherte Verdienst nicht nach Art. 24 Abs. 1 UVV. Vielmehr ist nach Absatz 4 für die neue Rente aus beiden Unfällen derjenige Lohn maßgebend, den der Versicherte im Jahr vor dem letzten Unfall bezogen hätte, wenn früher kein versicherter Unfall eingetreten wäre. Sinn und Zweck von Art. 24 Abs. 4 UVV ist es, für die revisionsweise Neufestsetzung der Invalidenrente nicht auf den in der Regel durch die Invalidität bereits reduzierten Lohn im Jahr vor dem Unfall abzustellen, sondern auf einen hypothetischen Lohn, den der Versicherte ohne Unfall hätte erzielen können. ${ }^{72}$

\section{Fragen zu den Komplementärrenten der Unfallversicherung}

\section{a) Festsetzung der Komplementärrente}

Wie bereits erwähnt ist beim Zusammentreffen von verschiedenen Renten die Invalidenversicherung primär leistungspflichtig. Die Unfallversicherung ergänzt diese Leistungen im Sinne einer bedingten Kumulation, d.h. die versicherte Person hat Anspruch auf beide Leistungen unter Berücksichtigung einer Überentschädigungsgrenze. In Abweichung von der allgemeinen Überentschädigungsregel in Art. 69 ATSG sieht das UVG in Art. 20 Abs. 2 bei der Koordination von Renten der Unfallversicherung mit Renten der AHV/IV ein besonderes Koordinationssystem vor, nach welchem die Komplementärrenten auf 90 Prozent des versicherten Verdienstes begrenzt sind.

Die Komplementärrente wird beim erstmaligen Zusammentreffen der erwähnten Renten festgesetzt und lediglich späteren Änderungen der für Familienangehörige be-

70 Ist ein Versicherter zu 70 Prozent als Behindertenbetreuer tätig, um daneben zu Hause die Matura für ein späteres Jura- oder Ökonomiestudium nachzuholen, und erhält er der Ausbildung wegen keinen geringeren Lohn, hat die Berechnung des versicherten Verdienstes nach Art. 22 Abs. 4 und nicht nach Art. 24 Abs. 3 UVV zu erfolgen (RKUV 2000 Nr. U 399 S. 379 f. E. 2b).

71 Art. 24 Abs. 3 UVV ist nicht anwendbar auf einen voll leistungsfähigen Versicherten, der aus irgendwelchen Gründen (z.B. mangelnder Einsatz, fehlende Leistungsfähigkeit oder bei einem ausländischen Staatsangehörigen allenfalls unzureichendes Anpassungsvermögen, Sprachschwierigkeiten oder im Vergleich zu schweizerischen Verhältnissen nicht gleichwertiges Wissen und Können) nicht den branchenüblichen Lohn erhält (BGE 106 V 229).

72 BGE 123 V 51 E. 3c; Art. 24 Abs. 4 UVV bezieht sich ausschliesslich auf Bezieher von Renten der Unfallversicherung (RKUV 1991 Nr. U 123 S. 152 E. 3a). Muss ein Versicherter infolge eines ersten Unfalls (und nicht infolge einer Wirtschaftskrise) seine Tätigkeit als Schlosser aufgeben und diejenige eines Handlangers aufnehmen, ist der Invalidität infolge eines weiteren Unfalls derjenige Lohn zu Grunde zu legen, den der Versicherte vor dem zweiten Unfall als Schlosser hätte verdienen können, wenn ihm der erste Unfall nicht zugestoßen wäre (EVGE 194122 E. 4). 
stimmten Teile der Rente der Invalidenversicherung oder der Alters- und Hinterlassenenversicherung angepasst. ${ }^{73}$

\section{b) Systematische Probleme des Zusammenspiels von Renten der Invalidenversiche-} rung und Komplementärrenten der Unfallversicherung

Das System der Komplementärrenten erscheint im Grundsatz als leicht verständlich, die Umsetzung in der Praxis ist jedoch mit diversen Problemen behaftet. Diese ergeben sich einerseits daraus, dass die Unfallversicherung ihre Rentenleistungen nach anderen Grundsätzen festlegt als die Invalidenversicherung. So berücksichtigt die Invalidenversicherung als finale Volksversicherung bei der Rentenbemessung auch Einschränkungen, die in der kausalen Unfallversicherung nicht beachtet werden (z.B. Einschränkung durch Krankheit).

Schwierigkeiten sind aber auch darauf zurückzuführen, dass auch bei der Berechnung der Komplementärrente das Kongruenzprinzip zu beachten ist, nach welchem nur Leistungen in die Überentschädigungsberechnung einbezogen werden dürfen, die in ereignisbezogener, personeller, sachlicher und zeitlicher Hinsicht kongruent sind. Bei der Ermittlung der Komplementärrente ist deshalb sicherzustellen, dass die Invalidenversicherungs- und die Unfallversicherungsrenten kongruente Tatbestände abdecken. Das bedeutet, dass die Unfallversicherung die von der Invalidenversicherung berücksichtigten unfallversicherungsfremden Anteile wie Krankheit, Haushalt, selbständige Erwerbstätigkeit ${ }^{74}$ und Alter ${ }^{75}$ auszusondern hat. ${ }^{76}$

Weitere Fragen stellen sich auch bei einer späteren Anpassung an veränderte Verhältnisse. Eine Anpassung der Komplementärrente erfolgt beim Dahinfallen oder Hinzukommen von Zusatz- und Kinderrenten der Alters-, Hinterlassenen- und Invalidenversicherung, bei einer Änderung der Berechnungsgrundlage einer solchen Rente und bei einer maßgeblichen Änderung des für die Unfallversicherung maßgebenden Invaliditätsgrades. ${ }^{77}$ Nach der ausdrücklichen Vorschrift in Art. 34 UVV führt auch eine Revision der Invalidenversicherungsrente zu einer Revision der UnfallversicherungsKomplementärrente. Dies unter dem Vorbehalt, dass die Revision der Invalidenversicherungsrente einen Faktor betrifft, der auch für die Unfallversicherungsrente maßgeblich ist, d.h. einen kongruenten Tatbestand.

Nachfolgend werden einzelne Konstellationen näher betrachtet.

73 Art. 20 Abs. 2 Satz 2 UVG.

74 Art. 32 Abs. 1 UVV.

75 Art. 28 Abs. 4 UVV.

76 Siehe Art. 32 Abs. 1 UVV: „Entschädigt eine Rente der IV auch eine nicht nach UVG versicherte Invalidität, wird bei der Berechnung der Komplementärrente nur jener Teil der Rente der IV berücksichtigt, welche die obligatorisch versicherte Tätigkeit abgilt.“

77 Art. 33 Abs. 2 lit. A-c UVV. 
Auswirkungen von nachfolgenden Änderungen im Rentenanspruch gegenüber der Alters-, Hinterlassenen und Invalidenversicherung: Art. 20 Abs. 2 und Art. 31 Abs. 4 UVG sehen vor, dass die Komplementärrente der Unfallversicherung beim erstmaligen Zusammentreffen mit der Alters-, Hinterlassenen- oder Invalidenrente festgesetzt wird und lediglich späteren Änderungen der für Familienangehörige bestimmten Teile der Rente oder den Änderungen im Bezügerkreis der Alters-, Hinterlassenen oder Invalidenversicherungsrente angepasst wird. Der Verordnungsgeber hat für die Komplementärrente zur Invalidenversicherungsrente konkretisierende Bestimmungen erlassen, ${ }^{78}$ welche auch für die Hinterlassenenrenten anwendbar sind. ${ }^{79}$ Diese Bestimmungen schließen auch die Anpassung wegen Änderung der Berechnungsgrundlagen ein, weshalb beim Splitting nach Art. $29^{\text {quinquies }}$ Abs. 3 AHVG, bei der Plafonierung gemäß Art. 35 AHVG, nach der Rechtsprechung aber auch bei der Ablösung der Witwen- durch eine Altersrente eine Anpassung der Komplementärrente erfolgt. ${ }^{80}$ Die Komplementärrente ist zudem auch anzupassen, wenn sich der für die Unfallversicherung maßgebende Invaliditätsgrad erheblich ändert. ${ }^{81}$ Tritt zu einer unfallbedingten Invalidität eine krankheitsbedingte Invalidität und wird die IV-Rente deswegen heraufgesetzt, hat dagegen keine Anpassung der Komplementärrente zu erfolgen.

Komplementärrente bei krankheits- und unfallbedingter Invalidität: Wenn eine Invalidenversicherungsrente auch eine krankheitsbedingte Invalidität entschädigt, wird bei der Berechnung der Komplementärrente nur derjenige Anteil der Invalidenversicherungsrente berücksichtigt, der die unfallbedingte Invalidität abgilt. ${ }^{82}$ Bei der betragsmäßigen Berechnung dieses Anteils ist zu bestimmen, welchen prozentualen Anteil die unfallbedingte Invalidität an der Gesamtinvalidität hat. Ein Beispiel: Die Invalidenversicherung richtet bei einem Invaliditätsgrad von 70 Prozent eine ganze Rente (= Rente von 100 Prozent) aus; die Unfallversicherung hat für ihren Bereich eine (unfallbedingte) Invalidität von 30 Prozent ermittelt. Zur Bestimmung des anrechenbaren Teils der Invalidenversicherungsrente stehen zwei Berechnungsmethoden zur Verfügung: Es kann als erste Methode - bestimmt werden, welchen Anteil die in der Unfallversicherung relevanten 30 Prozent an der ganzen Invalidität (100 Prozent) haben, was ebenfalls 30 Prozent ausmacht. Von der Invalidenversicherungsrente kann also die Unfallversicherung einen Anteil von 30 Prozent berücksichtigen. Es kann aber - als zweite Methode auch bestimmt werden, wie hoch der Anteil von 30 Prozent an 70 Prozent ist, was 42,8 Prozent ausmacht; die Unfallversicherung kann also 42,8 Prozent der Invalidenversicherungsrente berücksichtigen. Zutreffend erscheint die zweite der genannten Möglichkeiten; denn die Tatsache, dass bei einer Gesamtinvalidität von 70 Prozent eine Invalidenversicherungsrente von 100 Prozent ausgerichtet wird, ist eine Entscheidung des Invali-

78 Art. 33 UVV.

79 Art. 43 Abs. 6 UVV.

80 BGE 126 V 512.

81 Art. 33 Abs. 2 lit. c UVV.

82 Art. 32 Abs. 1 UVV. 
denversicherungsrechts, weshalb bei der Lösung der koordinationsrechtlichen Frage die Heranziehung der Referenzbasis von 100 Prozent unzutreffend erscheint.

Komplementärrente bei zusätzlich ausgeübter selbstständiger Erwerbstätigkeit: Die Unfallversicherung erfasst im Gegensatz zur Invalidenversicherung die selbstständige Erwerbstätigkeit nicht. Bei der Berechnung der Unfallversicherungs-Komplementärrente ist eine nicht obligatorisch in der Unfallversicherung versicherte Invalidität, d.h. derjenige Teil der Invalidenrente, der die selbständige Tätigkeit abdeckt, nicht zu berücksichtigen. ${ }^{83}$ Dies führt zu Schwierigkeiten, weil die Invalidenversicherung beide Tätigkeitsbereiche gemeinsam erfasst und die Invalidität - im Gegensatz zur gemischten Methode bei Teilerwerbstätigen - nicht getrennt ermittelt. Hier bleibt nur die Möglichkeit, die Rente in Berücksichtigung der vor dem Unfall erzielten Einkommen aufzuteilen.

Komplementärrente bei vorgerücktem Alter: Bei Personen im vorgerückten Alter bestimmt die Unfallversicherung den Invaliditätsgrad unter Berücksichtigung der Verhältnisse einer versicherten Person im mittleren Alter. ${ }^{84}$ Die Invalidenversicherung verzichtet auf eine Berücksichtigung des vorgerückten Alters, weshalb bei diesem Sozialversicherungszweig in der Regel ein höherer Invaliditätsgrad resultiert. In einem solchen Fall ist deshalb ebenfalls davon auszugehen, dass die Invalidenversicherung eine nicht obligatorisch unfallversicherte Invalidität im Sinne von Art. 32 Abs. 1 UVV entschädigt, was dazu führt, dass die Invalidenversicherungsrente bezüglich demjenigen Betrag, der sich aus dem entsprechend höheren Invaliditätsgrad ergibt, bei der Berechnung der Komplementärrente nicht berücksichtigt werden darf.

Komplementärrente im Alter: Wenn die versicherte Person das zum Bezug einer AHV-Altersrente maßgebende Alter erreicht, wird die ihr bisher ausgerichtete IV-Rente durch eine AHV-Altersrente ersetzt; demgegenüber richtet die UV die Invalidenrente weiterhin aus. ${ }^{85}$ Bei diesem Übergang wird die Komplementärrente der Unfallversicherung nicht neu berechnet; ${ }^{86}$ dies gilt aber dann nicht, wenn beim Übergang die Berechnungsgrundlagen ändern. ${ }^{87}$ Setzt die Unfallversicherungsrente erst nach Erreichen der Altersgrenze ein, so erfolgt eine Erhöhung der maßgebenden Grenze von 90 Prozent des

83 Art. 32 Abs. 1 UVV.

84 Art. 28 Abs. 4 UVV.

85 Art. 19 Abs. 2 UVG.

86 Art. 33 Abs. 1 UVV.

87 BGE 126 V 512, wo es um die Ablösung einer Witwenrente durch eine AHV-Altersrente ging; Art. 33 Abs. 2 lit. b UVV geht somit Art. 33 Abs. 1 UVV vor. In den seltenen Fällen, in welchen beim Übergang die AHV-Rente nicht nach den bisherigen, für die IV-Rente maßgebenden Berechnungsgrundlagen bestimmt wird, sondern aufgrund der bis zum Erreichen des AHV-Rentenalters geleisteten Beiträge neu und höher bestimmt wird, führt dies zum unbefriedigenden Ergebnis, dass die Unfallversicherung von den durch die versicherte Person nach Eintritt der Invalidität weiterhin geleisteten AHV-Beiträgen profitiert. 
versicherten Einkommens um die Altersrente. ${ }^{88}$ Anpassungen der so berechneten UVKomplementärrente sind in den Verordnungsbestimmungen nicht vorgesehen.

\section{Anpassung der Renten an die Teuerung}

Zum Ausgleich der Teuerung erhalten alle Bezügerinnen und Bezüger von Invalidenund Hinterlassenenrenten der Unfallversicherung Zulagen. Diese gelten als Bestandteil der Rente. ${ }^{89}$ Der Bundesrat setzt die Zulagen aufgrund des Landesindexes der Konsumentenpreise fest. Die Renten werden auf den gleichen Zeitpunkt wie die Renten der Alters- und Hinterlassenenversicherung der Teuerung angepasst. ${ }^{90}$ Die Teuerungszulagen werden aus Zinsüberschüssen und, soweit diese nicht ausreichen, nach dem Ausgabenumlageverfahren finanziert. ${ }^{91}$

\section{Besondere Problematik bei Teilzeitarbeit}

Da für alle vom Bundesgesetz über den Allgemeinen Teil der Sozialversicherung erfassten Sozialversicherungszweige die Invalidität nach dem gleichen Prinzip (Art. 16 ATSG) zu bestimmen ist, sollte auch in allen Zweigen derselbe Invaliditätsgrad resultieren. Die Rechtsprechung geht vom Grundsatz aus, dass mit Bezug auf den gleichen Gesundheitsschaden in den verschiedenen Sozialversicherungszweigen die Festlegung der Invalidität zum gleichen Ergebnis führen muss. ${ }^{92}$ Dieser Grundsatz führte die Rechtsprechung dazu, bestimmte Bindungswirkungen eines einmal getroffenen Entscheides zur Invaliditätsfestsetzung zu bejahen.

Diese Bindungswirkung findet bei manchen Sachverhalten eine Grenze. Besondere Schwierigkeiten ergeben sich bei Teilerwerbstätigen bezüglich der gegenseitigen Bindung der Versicherungsträger bei der Festlegung des Invaliditätsgrades. Bei teilweise Erwerbstätigen bemisst sich der Invaliditätsgrad nämlich nach der so genannten gemischten Methode. Dabei wird für die Teilerwerbstätigkeit die Invalidität nach dem Einkommensvergleich und die Invalidität im Aufgabenbereich nach der Methode des Betätigungsvergleichs festgelegt. Die Gesamtinvalidität wird nach Massgabe der zeitlichen Beanspruchung in den beiden Bereichen berechnet. ${ }^{93}$

Die Schwierigkeit besteht nun darin, dass in der Invalidenversicherung auch die Invalidität von Nichterwerbstätigen entschädigt wird. Im Gegensatz dazu wird in der Un-

\footnotetext{
88 Art. 32 Abs. 3 UVV.

89 Art. 34 Abs. 1 UVG.

90 Art. 34 Abs. 2 UVG.

91 Art. 90 Abs. 2 UVG.

92 BGE 119 V 470.

93 Art. 28 Abs. $2^{\text {ter }}$ IVG, ab 1. Januar 2008 Art. 28a Abs. 3 IVG.
} 
fallversicherung die Invalidität ausschliesslich im erwerblichen Bereich entschädigt. ${ }^{94}$ Folglich kann die Unfallversicherung nicht den durch die Invalidenversicherung festgesetzten Invaliditätsgrad mitberücksichtigen, falls dieser auch die Einschränkung im Aufgabenbereich erfasst. Es dürfen nur die sich im erwerblichen Bereich ergebenden Einschränkungen berücksichtigt werden.

Eine weitere Schwierigkeit ergibt sich bei Teilerwerbstätigen daraus, dass nach der Rechtsprechung im Bereich der Invalidenversicherung bei der Ermittlung des Invaliditätsgrades im erwerblichen Bereich bei Teilerwerbstätigen als hypothetisches Valideneinkommen nicht das Einkommen gilt, das der Teilerwerbstätige bei einer vollen Erwerbstätigkeit erzielen könnte, sondern das Einkommen, welches aus einem hypothetisch geleisteten Teilarbeitspensum als Gesunder resultieren würde. ${ }^{95}$ Die Unfallversicherung befolgt bei Teilerwerbstätigen eine gegenteilige Praxis, indem das Valideneinkommen auf einer hypothetischen Tätigkeit von 100 Prozent basiert. 96 Dies hat zur Folge, dass die Unfallversicherung weder den von der Invalidenversicherung berechneten Gesamtinvaliditätsgrad noch denjenigen für den erwerblichen Bereich übernehmen darf. Vielmehr hat sie den Invaliditätsgrad nach eigenen Kriterien zu ermitteln. ${ }^{97}$ Dass die verunfallte Person vor dem Unfall nur teilzeitlich erwerbstätig war, wirkt sich in der Unfallversicherung bei der Rentenhöhe aus, weil nur das Einkommen aus Teilerwerbstätigkeit versicherten Verdienst darstellt. 98

Bei teilinvaliden Personen stellt sich regelmäßig die Frage, wie bei der Leistungskoordination mit dem durch die betreffenden Personen allenfalls noch erzielbaren Resterwerb umzugehen ist. Die allgemeine Überentschädigungsregel in Art. 69 ATSG beantwortet die Frage nach der Berücksichtigung des Resterwerbes nicht. Die Materialien lassen erkennen, dass der Gesetzgeber davon ausgeht, dass eine Berücksichtigung eines zumutbarerweise erzielbaren Resterwerbes nur erfolgen kann, wenn eine entsprechende Regelung im Einzelgesetz eingefügt wird. ${ }^{99}$ Die Regelungen in den Einzelgesetzen sind diesbezüglich unterschiedlich. Von einer Berücksichtigung des nur zumutbarerweise erzielbaren Resterwerbseinkommens geht die Militärversicherung in Art. 32 Abs. 1 lit. c MVV 100 aus. Art. 14a ELV101 ermöglicht den Ergänzungsleistungsbehörden bei Teilinvaliden bestimmte, in der Verordnung festgesetzte Erwerbseinkommen anzurechnen.

\footnotetext{
94 Vgl. Art. 18 Abs. 1 UVG.

95 BGE $125 \mathrm{~V} 146$.

96 BGE $119 \mathrm{~V} 475 \mathrm{ff}$.

97 BGE 119 V $475 \mathrm{ff}$.

98 Vgl. Art. 15 Abs. 2 UVG.

99 Kieser (Fn. 58), Art. 69 Rz. 23.

100 Verordnung vom 10. November 1993 über die Militärversicherung.

101 Verordnung vom 15. Januar 1971 über die Ergänzungsleistungen zur Alters-, Hinterlassenen- und Invalidenversicherung.
} 
In der Unfallversicherung wird demgegenüber nur das tatsächlich erzielte Erwerbseinkommen angerechnet, wie dies in Art. 51 Abs. 3 UVV vorgesehen ist. ${ }^{102}$

\section{Zusammenfassende Würdigung}

Fasst man nun diesen kurzen Überblick über die schweizerische Unfallversicherung und das Bemessungssystem ihrer Geldleistungen zusammen, so ergibt sich das folgende Gesamtbild:

Im Wesentlichen hat sich die gesetzliche Ordnung bewährt. Die gegenwärtige Finanzierung darf als solid bezeichnet werden.

Soweit ersichtlich bereitet die unterschiedliche Finanzierungsweise der Taggelder und anderer kurzfristiger Leistungen durch das Umlageverfahren und der Rentenleistungen durch das Umlagedeckungsverfahren (als Kapitaldeckungselement innerhalb der Unfallversicherung) keine nennenswerten Probleme.

Im Hinblick auf die demographische Entwicklung erscheint die mit dem Umlagedeckungsverfahren für Renten erzielte Entlastung der künftigen Generation als wünschenswert. Sie kann als Ausdruck horizontaler Solidarität innerhalb derselben Generation gelten, d.h. als Ausdruck der Solidarität der gegenwärtig beitragsbelasteten Arbeitgeber untereinander.

Die Invalidenrenten der schweizerischen Unfallversicherung sind nur darauf ausgerichtet, den Erwerbsausfall für eine nach der Eingliederung verbleibende Einschränkung in der Erwerbsfähigkeit zu kompensieren. Selbst schwere körperliche Beeinträchtigungen führen damit zu keinen Rentenleistungen der Unfallversicherung, wenn sie keine unmittelbaren Auswirkungen auf die Erwerbsfähigkeit der versicherten Person haben.

102 In der bis 31.12.2004 gültigen Fassung sah Art. 24 Abs. 2 Satz 2 BVV 2 vor, dass den Bezügern von Invalidenleistungen das weiterhin erzielte Erwerbseinkommen angerechnet werden kann. In BGE $123 \mathrm{~V} 88 \mathrm{f}$. hat des Eidgenössische Versicherungsgericht festgehalten, dass gemäß dieser Rechtsgrundlage nur das effektiv erzielte, nicht aber das zumutbarerweise erzielbare Erwerbseinkommen angerechnet werden könne. So konnte eine versicherte Person, die infolge eines Unfalls zu 55 Prozent invalid war, mit einer UVG-Invalidenrente, mit einer halben Rente der Invalidenversicherung und einer 55 prozentigen Invalidenrente der Vorsorgeeinrichtung ein Gesamtrenteneinkommen von fast 90 Prozent des mutmaßlich entgangenen Bruttoverdienstes erreichen, ohne sich darum bemühen zu müssen, ihre Resterwerbsfähigkeit mit einer leidensangepassten Arbeit noch zu nutzen. Diesem Entscheid ist in der Literatur verschiedentlich Kritik erwachsen. Mit Wirkung ab 1. Januar 2005 hat der Bundesrat Art. 24 Abs. 2 BVV 2 dahingehend geändert, dass den „Bezügern von Invalidenleistungen (...) überdies das weiterhin erzielte oder zumutbarerweise noch erzielbare Erwerbs- oder Ersatzeinkommen angerechnet" wird. Mit dieser Neuregelung soll vermieden werden, dass die Vorsorgeeinrichtungen Leistungen für nicht invaliditätsbedingten Erwerbsausfall erbringen müssen. Zudem sollen diejenigen Teilinvaliden, welche freiwillig keiner Teilerwerbstätigkeit nachgehen, finanziell nicht mit denjenigen Teilinvaliden, die ihre Restarbeitsfähigkeit verwerten, gleichgestellt werden. Es ist aber darauf hinzuweisen, dass diese Regel nicht unbedingt praktikabel ist und ebenfalls in berechtigter Kritik steht. 
Dem Ausgleich des immateriellen Körperschadens, der nach einem Unfall noch verbleibt, dient die Integritätsentschädigung. Es handelt sich dabei um eine gesetzlich geordnete Form der Genugtuung (Schmerzensgeld), die gegenwärtig auch für schwerste Beeinträchtigungen auf eine einmalige Summe von 126'000 Franken beschränkt ist.

Die juristisch-praktischen Probleme des schweizerischen Unfallrentensystems liegen bei der Schnittstelle zwischen der Invalidenrente der Invalidenversicherung und der Komplementärrente der Unfallversicherung. Für viele mögliche Problemkonstellationen hat die Praxis indes praktikable Lösungen entwickelt.

Das unterschiedliche Leistungsniveau von Kranken- und Unfallversicherung, insbesondere die weitgehend fehlenden Geldleistungen in der Krankenversicherung, bilden immer wieder Anlass zu rechtspolitischer Kritik. In jüngerer Zeit sorgte ein Vorschlag für Diskussionen, der - in Anlehnung an das niederländische System - die Abschaffung der Unfallversicherung in ihrer gegenwärtigen Form fordert. 103 Sozialpolitisch sei es nicht haltbar, dass es für die Betroffenen einen derart erheblichen Unterschied ausmacht, ob eine Invalidität wegen Unfall (was vorteilhafter ist) oder wegen Krankheit eintritt. Mag der formulierte Anstoss vorerst auch noch utopisch anmuten, so werden die mit ihm verfolgten sozialpolitischen Anliegen hoffentlich dennoch auf fruchtbaren Boden fallen.

103 Olivier Steiner, Die Abschaffung der Unfallversicherung. Eine Untersuchung zur Ungleichbehandlung von Unfall und Krankheit im schweizerischen und niederländischen Sozialrecht, Zü$\mathrm{rich} / \mathrm{Basel} / \mathrm{Genf} 2007$. 


\title{
Reformbestrebungen der Rentenbemessung in der deutschen gesetzlichen Unfallversicherung
}

\author{
Dr. Andreas Kranig
}

\section{Einführung}

Die letzte größere Reform des Unfallversicherungsrechts in Deutschland liegt über zehn Jahre zurück. Damals wurde die Gesetzliche Unfallversicherung durch das Unfallversicherungs-Einordnungsgesetz als Siebtes Buch in das Sozialgesetzbuch eingeordnet. Schon damals gab es gewichtige Stimmen aus der Wissenschaft - ich nenne nur Herrn Professor Gitter -, die das Prinzip der abstrakten Schadensbemessung im Rentenrecht der gesetzlichen Unfallversicherung in Zweifel zogen. Ein Übergang zu einer konkreteren Schadensbemessung mit expliziter Berücksichtigung des Erwerbsschadens und des immateriellen Schadens wurde damals - vor etwa 13 Jahren - intern im damaligen Bundesministerium für Arbeit und Sozialordnung geprüft. Eine grundlegende Erneuerung des Rentenrechts wurde damals aber verworfen. Ich sehe hierfür vier Gründe:

- Die gesetzestechnischen Schwierigkeiten für eine Umsetzung der konkreten Schadensbemessung erschienen zu groß.

- Weder in der Wissenschaft noch unter den Praktikern der Gesetzlichen Unfallversicherung fanden sich mehrheitlich Befürworter einer konkreten Schadensbemessung.

- Vor allem aber fehlte es am politischen Druck - sei es von den Sozialpartnern, sei es aus den politischen Parteien -, eine Änderung in Angriff zu nehmen.

- Schließlich entsprach es dem Kalkül im damaligen Gesetzgebungsverfahren, grundlegende und damit politische umstrittene Reformen nicht mit der Einordnung des Unfallversicherungsrechts in das Sozialgesetzbuch zu verbinden. Für den Fall einer tiefergreifenden Reform wurde befürchtet, dass das Vorhaben scheitern oder jedenfalls ins Stocken geraten könnte.

\section{Gesetzgebungsverfahren zum Unfallversicherungs-}

\section{Reformgesetz (UVRG)}

Seitdem haben sich die Zeiten geändert. Betrachtet man die Entwicklungen der letzten Jahre, so sind um die gesetzliche Unfallversicherung herum alle anderen Sozialversicherungszweige mehrfach und tiefgreifend reformiert worden. Die Sozialpolitik muss- 